\title{
Hydrodynamic Synchronization and Metachronal Waves on the Surface of the Colonial Alga Volvox carteri
}

\author{
Douglas R. Brumley, Marco Polin, Timothy J. Pedley, and Raymond E. Goldstein \\ Department of Applied Mathematics and Theoretical Physics, Centre for Mathematical Sciences, University of Cambridge, \\ Wilberforce Road, Cambridge CB3 OWA, United Kingdom \\ (Received 5 August 2012; published 27 December 2012)
}

\begin{abstract}
From unicellular ciliates to the respiratory epithelium, carpets of cilia display metachronal waves, longwavelength phase modulations of the beating cycles, which theory suggests may arise from hydrodynamic coupling. Experiments have been limited by a lack of organisms suitable for systematic study of flagella and the flows they create. Using time-resolved particle image velocimetry, we report the discovery of metachronal waves on the surface of the colonial alga Volvox carteri, whose large size and ease of visualization make it an ideal model organism for these studies. An elastohydrodynamic model of weakly coupled compliant oscillators, recast as interacting phase oscillators, reveals that orbit compliance can produce fast, robust synchronization in a manner essentially independent of boundary conditions, and offers an intuitive understanding of a possible mechanism leading to the emergence of metachronal waves.
\end{abstract}

PACS numbers: 87.16.Qp, 05.45.Xt, 47.63.-b, 87.18.Tt

A major evolutionary breakthrough for unicellular organisms was the incorporation of appendages for selfpropulsion. In ancestral eukaryotes, these took the form of 10-20 $\mu \mathrm{m}$ long hairlike motile organelles known as flagella which, together with their shorter ciliary version, are now common to most extant eukaryotic species. Whether in ciliates like Paramecium or the lining of the respiratory tract, cilia and flagella usually appear in large groups within which their individual motions tend to synchronize in patterns known as metachronal waves (MWs), modulations of the beating phase on the scale of many undulipodia [1]. Many theoretical studies [2-4] hint at a higher efficiency of propulsion by MWs, and suggest a possible selective advantage of mutations that stabilize their occurrence. Yet, the mechanisms behind their emergence and the selection of wave patterns are still unclear.

Early reports of correlated changes in the directions of flagellar beating and MW propagation [5] led to the hypothesis of a mechanical origin for synchronization, and motivated theoretical studies involving hydrodynamically coupled filaments driven by various types of internal engines [6-9]. While these models show a general tendency towards metachronal coordination and help explain the generation and regulation of flagellar motion [10], their complexity prevents a simple understanding of the mechanism responsible for synchronization.

Minimal models of hydrodynamically coupled selfsustained oscillators with few degrees of freedom give insight into the emergence of MWs, and such

Published by the American Physical Society under the terms of the Creative Commons Attribution 3.0 License. Further distribution of this work must maintain attribution to the author(s) and the published article's title, journal citation, and DOI. synchronization has been investigated experimentally with rotating paddles [11], microrotors [12], and colloids in optical tweezers $[13,14]$, in simulations of rotating helices [15], and in several theoretical models of spheres driven along trajectories, either fixed [16-21] or flexible [22]. We now know that under certain circumstances $[17,21]$ hydrodynamics alone can phase lock two simple oscillators. Whether this mechanism can account for the rapid and robust synchronization seen in biological systems [23] remains unclear, although such behavior does occur when there is flexibility in the oscillators' trajectories [22]. Recent experimental evidence [24] is consistent with the hypothesis of orbit flexibility and hydrodynamic coupling as a possible origin of flagellar synchronization. At the same time, synchronization of an isolated pair is not sufficient to guarantee metachronism of an ensemble, highlighting the need to understand better the link between local and global coordination. Progress hinges on the identification of suitable experimental systems to study MWs and test the intuition developed from theory, a goal which remains unfulfilled.

Here we introduce the colonial green alga Volvox carteri [25] as a platform to study coordination in ensembles of eukaryotic flagella. Volvox is composed of thousands of biflagellate somatic cells embedded in a spherical extracellular matrix of radius $R \sim 200 \mu \mathrm{m}$, beating towards the colony posterior with a nearly planar waveform. We report the existence of MWs in this simple, readily observable and easily manipulable system, and describe a straightforward method to quantify them. The flagella of Volvox are spaced relatively far apart compared to the celebrated ciliate Paramecium, and hence they are more nearly in the weak-coupling limit amenable to theory. A minimal elastohydrodynamic model describes these MWs semiquantitatively, and confirms that elasticity can 
lead to robust synchronization. The model proposes a simple mechanism for the spontaneous choice of MW direction, and provides a unifying view of the synchronization patterns observed by previous studies in finite chains of oscillators.

Growth conditions for Volvox carteri f. nagariensis (strain EVE) have been described previously [26]. Individual colonies were inserted in a $25 \times 25 \times 5 \mathrm{~mm}$ observation chamber filled with fresh medium [27] seeded at $2 \times 10^{-4}$ volume fraction with $0.5 \mu \mathrm{m}$ polystyrene microspheres (Invitrogen, USA). Colonies were captured, oriented, and held using two micropipettes with $100 \mu \mathrm{m}$ diameter tips, hosted on pipette holders (World Precision Instruments, USA) held by motorized micromanipulators (Patchstar, Scientifica, UK), and connected to two manual microinjectors (Sutter Instruments Co., USA). Custommade rotation stages mounted on each holder allowed rotation around the pipette's axis [24]. We aligned the axis of each colony with the focal plane of a $40 \times$ Plan Fluor objective (NA 0.6) on a Nikon TE2000-U inverted microscope, and recorded $30 \mathrm{~s}$ long movies of the surrounding flow with a high-speed video camera (Fastcam SA3, Photron, USA) at 500 fps under bright field illumination. A long pass interference filter with a $10 \mathrm{~nm}$ transition ramp centered at $620 \mathrm{~nm}$ (Knight Optical, U.K.) prevented phototactic responses. The projection of the velocity field $\boldsymbol{u}$ onto the focal plane was reconstructed using an open source particle image velocimetry [28] toolbox for Matlab (MatPIV), and decomposed into polar coordinates $\boldsymbol{u}=u_{r} \boldsymbol{e}_{r}+u_{\theta} \boldsymbol{e}_{\theta}$ as in Fig. 1(c).

For each Volvox, the radial component $u_{r}\left(r^{*}, \theta, t\right)$ at $r^{*}=1.3 R$ [Fig. 1(c)] is used to resolve spatially the beating phase of the somatic cells along the colony's meridian opposite to the holding pipette. As shown in Fig. 1(a) kymographs of $u_{r}\left(r^{*}, \theta, t\right)$ reflect clearly the propagation of a MW from the anterior towards the posterior of the colony. This is a symplectic $\mathrm{MW}$, propagating in the same direction as the flagellar power stroke. Inspection of several colonies at different orientations confirms that the wave is mostly symplectic with only a minimal diaplectic (lateral) component. The wave parameters can be determined from the normalized correlation function

$$
C(\Delta \theta, \Delta t)=\frac{\left\langle\bar{u}_{r}\left(r^{*}, \theta+\Delta \theta, t+\Delta t\right) \bar{u}_{r}\left(r^{*}, \theta, t\right)\right\rangle}{\left\langle\bar{u}_{r}\left(r^{*}, \theta, t\right)^{2}\right\rangle},
$$

where $\overline{\boldsymbol{u}}(r, \theta, t)$ is the deviation of the measured flow from its time average, and \langle\rangle denote averaging over $t$ and $\theta$. Fitting the correlation function as $C(\Delta \theta, \Delta t)=$ $e^{-\Delta t / \tau} e^{-\Delta \theta / L} \cos (k \Delta \theta-\omega \Delta t)$ yields the average beat frequency $f=1 / T=\omega / 2 \pi$, autocorrelation decay time $\tau$, and wave number $k$ for each colony. Averaging over 60 colonies we obtain $f=32.3 \pm 3.1 \mathrm{~Hz}, \tau / T=3.4 \pm 2.0$, $L=0.35 \pm 0.10$, the correlation length, and $k=4.7 \pm$ 0.9 , with individual data summarized in Figs. 1(d)-1(f) [29]. The small value of $\tau / T$ is surprising. Its origin is likely a combination of noisy flagellar dynamics [24] and collective phase slips, and will be addressed elsewhere. We emphasize that $k>0$ for all colonies, indicating that despite the absence of any direct connections between somatic cells symplectic MWs are ubiquitous.

We now turn to a model for MWs. Inspired by the orbits of the flagellar tips of Volvox somatic cells [Fig. 2(a)], we generalize previous studies $[17,18,22]$ and model the synchronous flagellar pair of an individual cell as a sphere of radius $a$ elastically bound to a circular orbit of radius $r_{0}$,
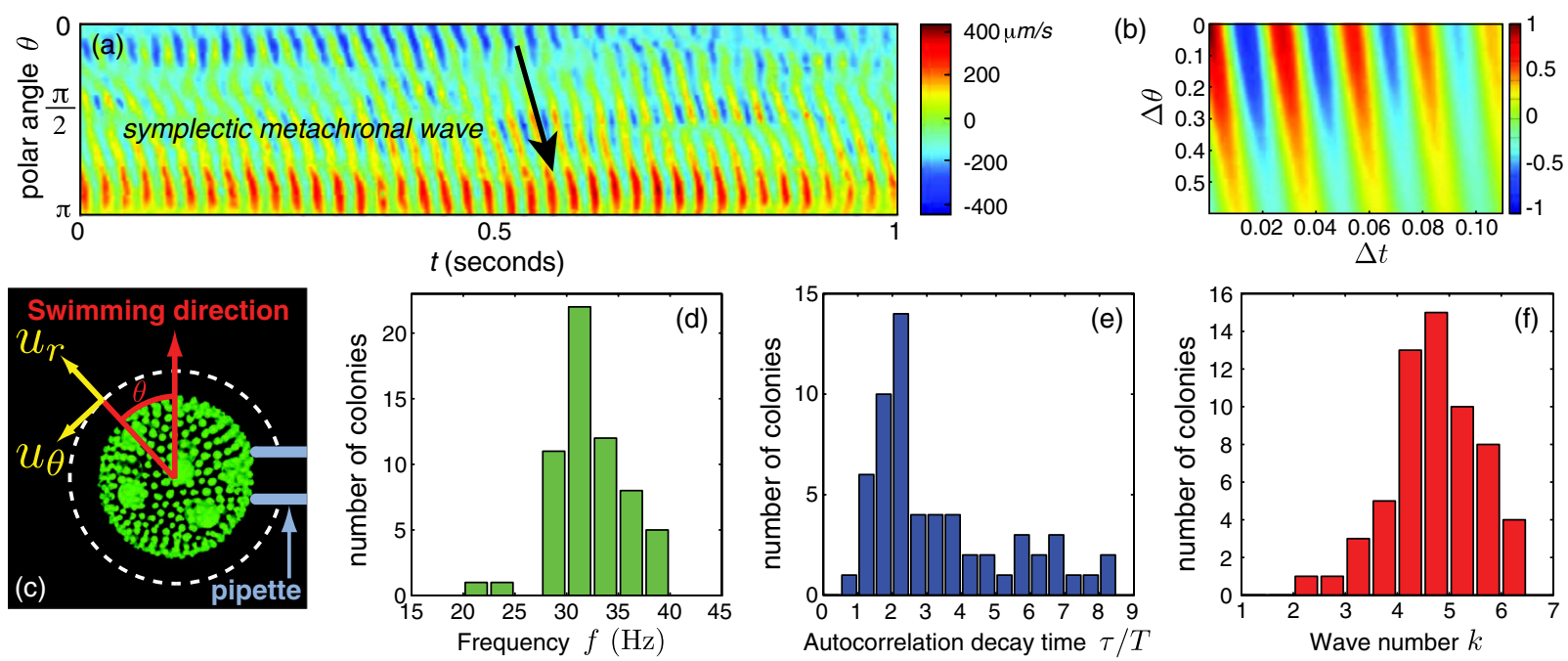

FIG. 1 (color online). Metachronal waves of Volvox. (a) Radial component $u_{r}(\theta, t)$ of the flow, measured at $r^{*}=1.3 R$. (b) Correlation function $C(\Delta \theta, \Delta t)$ in Eq. (1). (c) Confocal image of Volvox, showing somatic cells (small dots), interior daughter colonies (large circles), colonial axis, and radius for velocity components (dashed). Histograms of beat frequencies (d), decay times of $C(0, \Delta t)$ scaled by beat period (e), and metachronal wave number (f) for 60 colonies. 

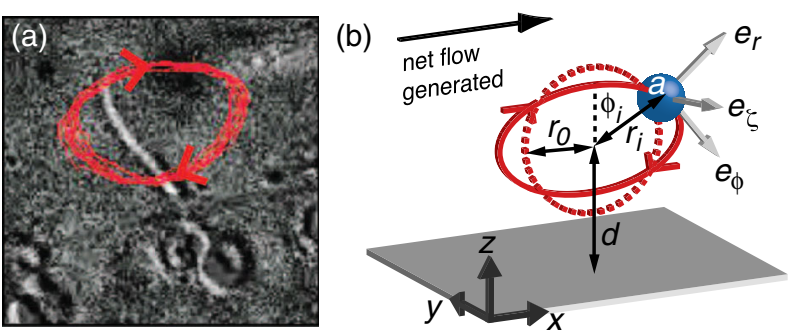

FIG. 2 (color online). Modeling flagella. (a) Tip trajectory over 4 beats of a flagellum of a Volvox somatic cell. (b) Model flagellum: a sphere of radius $a$ elastically bound to a circular trajectory of radius $r_{0}$ (dashed) perpendicular to a no-slip plane, driven by a constant tangential force.

perpendicular to a no-slip plane, with the center at $\boldsymbol{x}^{0}=$ $\left(x^{0}, y^{0}, d\right)$ [Fig. 2(b)]. The sphere is driven by a constant tangential force $f^{\mathrm{dr}} \boldsymbol{e}_{\phi}$, and its velocity $\boldsymbol{v}$ follows the forcebalance requirement of Stokes flow:

$$
\boldsymbol{\gamma}(\boldsymbol{x}) \cdot \boldsymbol{v}=-\lambda\left(r-r_{0}\right) \boldsymbol{e}_{r}-\eta \zeta \boldsymbol{e}_{\zeta}+f^{\mathrm{dr}} \boldsymbol{e}_{\phi} .
$$

Here the spring stiffnesses $\lambda$ and $\eta$ in the radial $\left(\boldsymbol{e}_{r}\right)$ and transverse $\left(\boldsymbol{e}_{\zeta}\right)$ directions determine the deformation of the trajectory in response to external stresses. The friction tensor $\boldsymbol{\gamma}(\boldsymbol{x})=\gamma_{0}\left[\mathbf{I}+(9 a / 16 z)\left(\mathbf{I}+\boldsymbol{e}_{z} \boldsymbol{e}_{z}\right)+\mathcal{O}\left((a / z)^{3}\right)\right]$ [30] depends on the sphere's position $\boldsymbol{x}=\boldsymbol{x}^{0}+$ $(r \sin \phi, 0, r \cos \phi)$, and $\gamma_{0}=6 \pi \mu a$ is the drag on the sphere in an unbounded fluid of viscosity $\mu$. Because the drag is nonisotropic, the sphere deviates slightly from the prescribed circle $\left(\delta r / r_{0} \sim 0.5 \%\right.$ here). For each set of parameters studied we simulate an isolated sphere, and recover the limit cycle $(r(t), \phi(t), \zeta(t))$ and the orbital period $T$, from which we define the phase of the oscillator $\Phi=\Phi(\phi)$ where $\dot{\Phi}=\omega=2 \pi / T[18,31]$, which is used to study the coupled dynamics of such spheres. The sphere's height above the wall defines distal and proximal sections of the orbit which mimic the natural power and recovery strokes of the flagellum. As expected [17] this asymmetry induces a net flow in the direction of the power stroke.

For $N$ identical oscillators at instantaneous positions $\left\{\boldsymbol{x}_{i}\right\}$ the hydrodynamic drag on the $i$ th sphere is $-\boldsymbol{\gamma}\left(\boldsymbol{x}_{i}\right) \cdot\left[\boldsymbol{v}_{i}-\right.$ $\left.\sum_{j \neq i} \mathbf{G}\left(\boldsymbol{x}_{j}, \boldsymbol{x}_{i}\right) \cdot \boldsymbol{F}_{j}^{\text {ext }}\right]$, where $\boldsymbol{F}_{j}^{\text {ext }}$ is the net external force acting on the $j$ th sphere [right-hand side of Eq. (2)]. $\mathbf{G}\left(\boldsymbol{x}_{i}, \boldsymbol{x}_{j}\right)$ is the Green's function ("Blake tensor") in the presence of a no-slip wall [32], which decays like $r^{-3}$ for displacements parallel to the wall instead of the characteristic $r^{-1}$ for a point force in bulk. The time evolution of the system of $N$ moving spheres is then given by

$$
\left[\mathbf{R}^{T} \mathbf{M}^{-1} \mathbf{\Gamma R}\right]_{j i}\left(\begin{array}{c}
r_{i} \dot{\phi}_{i} \\
\dot{r}_{i} \\
\dot{\zeta}_{i}
\end{array}\right)=\left(\begin{array}{c}
f^{\mathrm{dr}} \\
-\lambda\left(r_{j}-r_{0}\right) \\
-\eta \zeta_{j}
\end{array}\right) .
$$

The $3 N \times 3 N$ matrices $\boldsymbol{\Gamma}, \mathbf{M}$, and $\mathbf{R}$ are defined in terms of their constitutive $3 \times 3$ blocks $\Gamma_{i j}=\delta_{i j} \gamma\left(x_{i}\right), \quad \mathbf{M}_{i j}=$ $\delta_{i j} \mathbf{I}+\left(1-\delta_{i j}\right) \boldsymbol{\gamma}\left(\boldsymbol{x}_{i}\right) \cdot \mathbf{G}\left(\boldsymbol{x}_{j}, \boldsymbol{x}_{i}\right)$, and $\mathbf{R}_{i j}=\delta_{i j} \mathbf{R}_{i}$, where the columns of the rotation $\mathbf{R}_{i}$ are given by the vectors $\boldsymbol{e}_{\phi_{i}}$, $\boldsymbol{e}_{r_{i}}$, and $\boldsymbol{e}_{\zeta_{i}}$, respectively.

Taking advantage of the fact that MWs in Volvox are almost exactly symplectic, we represent the $\sim 30$ somatic cells along a colony's meridian as an open-ended linear chain of model oscillators. Their orbits are centered at $\boldsymbol{x}_{i}^{0}=(i \ell, 0, d)$, regularly spaced a distance $\ell>0$ apart, and the driving force $f^{\mathrm{dr}}>0$ turns them with an angular speed along the positive $y$ axis. In this configuration, the choice of $\eta$ does not affect the dynamics. We explore the behavior of chains of 2-50 oscillators, with $r_{0} / d=0.5$, $2 \leq \ell / d \leq 10$, and spring stiffnesses $0.01<\lambda d / f^{\mathrm{dr}}<10$. Within this parameter range the system evolves to a steady state profile in a few tens or hundreds of beating cycles, regardless of initial conditions. This phase-locking time scale increases with increasing stiffness of the orbit, and eventually diverges for $\lambda \rightarrow \infty$ (holonomic constraint), in line with previous studies of geometrically constrained oscillators [17]. The phase profiles after $t / T=1200$ beats for 30 interacting spheres, representative of the general behavior, are summarized in Fig. 3. For stiff springs the phase propagates outwards from the center of the chain, creating a chevron pattern previously encountered in similar systems [22,33], but as $\lambda$ decreases the phase-locked pattern morphs into a symplectic MW $\left(\Phi_{i}<\Phi_{i-1} \forall i\right)$. For these values of $\lambda$, we verified that $2 \mathrm{D}$ arrays display symplectic MWs, with wave numbers almost identical to the corresponding 1D configurations. The ability to generate a robust MW in an open-ended chain, which mimics natural boundary conditions for ciliary arrays, is indeed a necessary requirement for any reasonable model of flagellar coordination.

To represent Volvox flagella, we use their bending rigidity $\kappa=4 \times 10^{-22} \mathrm{Nm}^{2}$ [22] and length $L \simeq 10 \mu \mathrm{m}$, to estimate a radial spring stiffness $\lambda \simeq 4 \times 10^{-7} \mathrm{Nm}^{-1}$. Approximating the driving force as $f^{\mathrm{dr}}=2 \pi r_{0} \gamma_{0} / T$, and taking $a \sim 1 \mu \mathrm{m}, T \sim 1 / 33 \mathrm{~s}$, and $d / r_{0} \sim 1$, we obtain $\lambda d / f^{\mathrm{dr}} \sim 0.1$. This value is indeed situated in the symplectic MW regime of Fig. 3. A linear array of these oscillators

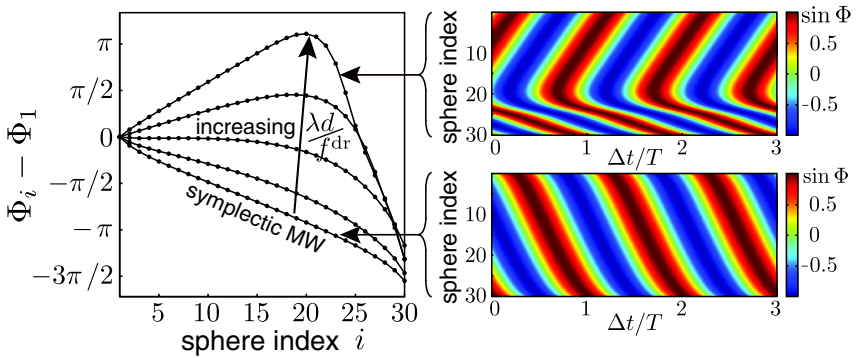

FIG. 3 (color online). Phase profile of an array of 30 spheres after $t / T=1200$ beats. Results are shown for $\lambda d / f^{\mathrm{dr}}=0.1,0.5$, $1,1.5,2$, and with $r_{0} / d=0.5, l / d=2, a / d=0.01$. 

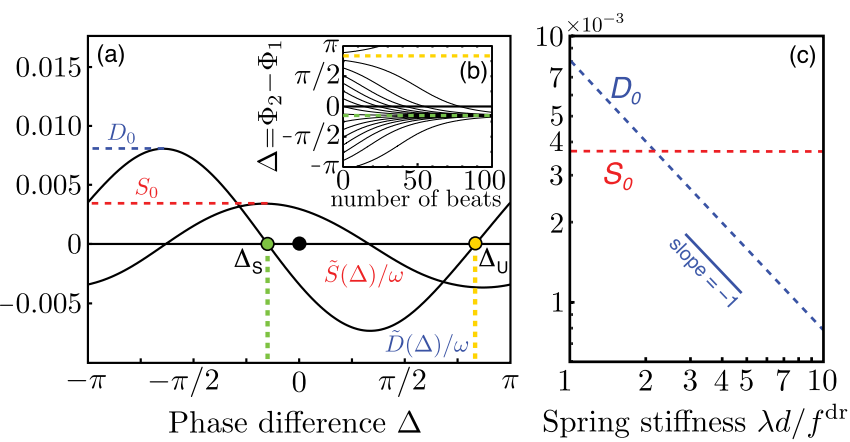

FIG. 4 (color online). Phase oscillator reduction. (a) Coupling functions $\tilde{D}(\Delta)$ and $\tilde{S}(\Delta)$ for a pair of interacting spheres. We have used the parameters $r_{0} / d=0.5, l / d=2, a / d=0.01$, $\lambda d / f^{\mathrm{dr}}=1$. (b) Phase difference, $\Delta=\Phi_{2}-\Phi_{1}$, for various initial conditions. (c) Amplitude of coupling functions for different spring stiffnesses.

spaced $l / d=2$ apart develops a MW with wave number $k \simeq 2$, which agrees reasonably well with the experimental values presented in Fig. 1(f).

The dynamics of the chain of oscillators can be understood by studying the case $N=2$. The interaction between two spheres induces only small perturbations around their limit cycles, and the coupled system can be described by the evolution of the phase difference $\dot{\Delta}=\dot{\Phi}_{2}-\dot{\Phi}_{1}=$ $\omega^{-}+D(\Delta, \Sigma)$ and sum $\dot{\Sigma}=\dot{\Phi}_{2}+\dot{\Phi}_{1}=\omega^{+}+S(\Delta, \Sigma)$, where $D$ and $S$ are effective coupling terms, and $\omega^{ \pm}=$ $\omega_{2} \pm \omega_{1}$. For sufficiently stiff springs, the phase difference evolves much more slowly than the sum, and we can average over a cycle of $\Sigma$ to obtain $\dot{\Delta}=\omega^{-}+\tilde{D}(\Delta)$ and $\dot{\Sigma}=\omega^{+}+\tilde{S}(\Delta)$. The functions $\tilde{D}$ and $\tilde{S}$ [Fig. 4(a)] are calculated numerically by simulating the system for many initial conditions [Fig. 4(b)]. Over the parameter range studied we consistently find a stable equilibrium point at a negative phase lag $\Delta_{S}<0$, and concurrently an increase in the average beating frequency $\left(\tilde{S}\left(\Delta_{S}\right)>0\right)$, a consequence of the lower drag at synchrony $[15,22]$. Nontrivial phase-locking results from the interaction between the wall-modified flows, the compliance of the orbits, and the influence of deformations on phase speed. The flow components perpendicular to the wall modify the in-phase configuration that would be stable in an unbounded fluid [22] by compressing slightly the orbit of the upstream sphere, which speeds up, and dilating the orbit of the downstream one, which slows down. The stable equilibrium is then skewed to a negative phase lag. The observed phase locking is robust against perturbations in orientation and relative position of the trajectories, or the use of more general driving forces.

Figure 4(c) shows the characteristic dependence of the amplitudes $D_{0}$ and $S_{0}$ of the effective potentials on the spring constant $\lambda$, from which we recognize $\operatorname{stiff}\left(D_{0}<S_{0}\right)$ and $\operatorname{soft}\left(D_{0}>S_{0}\right)$ regimes. Although they do not influence phase-locking for an isolated pair, these regimes lead to different steady states in an open chain [Fig. 3]. Drag reduction induced by synchronization is more pronounced on interior oscillators within the chain, which will tend to lead, creating in the stiff regime a chevron steady-state phase profile. However, in the soft regime, where the drive towards a nonzero phase lag $\Delta_{S}$ is strong $\left(D_{0}>S_{0}\right)$, cooperative speeding will only cause small perturbations around $\Delta_{S}$, and neighboring oscillators will phase lock with lags of the same sign as $\Delta_{S}$. In the present case this generates a symplectic MW $\left(\Phi_{i}<\Phi_{i-1} \forall i\right)$. For systems in which $\Delta_{S}=0$ [22], arbitrarily small perturbations can skew the phase lag to either positive or negative values, and this precludes the emergence of a well-defined MW. We also note that $\Delta_{S}$ is independent of $\lambda$, and scales as $\sim \ell^{-1}$ for large $\ell / d$. The amplitudes of the coupling functions scale according to $D_{0}, S_{0} \sim \ell^{-3}$ as expected from hydrodynamics.

The observation of MWs in the green alga Volvox carteri shows that large scale flagellar coordination is possible even in weakly coupled systems, and that detailed quantitative information on the average wave properties can be obtained with modern fluid dynamical techniques. The manner in which these properties depend on geometric factors such as the intercellular spacing and flagellar length will be addressed elsewhere. The model discussed here, with an interplay between wall-modified flows, orbit compliance, and deformation-induced changes in phase speed, reproduces the coordination of Volvox somatic cells in an open ended chain, which resembles a finite size array of flagella. This simple model may thus prove useful in clarifying the diverse types of MWs observed in nature. More generally, the question of whether orbital compliance is a necessary condition for metachronism will need to be addressed if we are to understand the origins of eukaryotic flagellar synchronization.

We are grateful to I. Tuval and A. I. Pesci for illuminating discussions. This work was supported by The Gates Cambridge Trust (D.R.B.), an EPSRC Postdoctoral Fellowship (M.P.), the BBSRC, and ERC Advanced Investigator Grant 247333 (R. E. G.).

[1] E. W. Knight-Jones, Q. J. Microsc. Sci. 95, 503 (1954).

[2] S. Gueron and K. Levit-Gurevich, Proc. Natl. Acad. Sci. U.S.A. 96, 12240 (1999).

[3] S. Michelin and E. Lauga, Phys. Fluids 22, 111901 (2010).

[4] N. Osterman and A. Vilfan, Proc. Natl. Acad. Sci. U.S.A. 108, 15727 (2011).

[5] C. Brennen and H. Winet, Annu. Rev. Fluid Mech. 9, 339 (1977).

[6] K. E. Machin, Proc. R. Soc. B 158, 88 (1963).

[7] S. Gueron, K. Levit-Gurevich, N. Liron, and J. J. Blum, Proc. Natl. Acad. Sci. U.S.A. 94, 6001 (1997).

[8] B. Guirao and J.-F. Joanny, Biophys. J. 92, 1900 (2007). 
[9] Y. Yang, J. Elgeti, and G. Gompper, Phys. Rev. E 78, 061903 (2008).

[10] I. H. Riedel-Kruse, A. Hilfinger, J. Howard, and F. Jülicher, HFSP J. 1, 192 (2007).

[11] B. Qian, H. Jiang, D. A. Gagnon, K. S. Breuer, and T. R. Powers, Phys. Rev. E 80, 061919 (2009).

[12] R. Di Leonardo, A. Búzás, L. Kelemen, G. Vizsnyiczai, L. Oroszi, and P. Ormos, Phys. Rev. Lett. 109, 034104 (2012).

[13] J. Kotar, M. Leoni, B. Bassetti, M. C. Lagomarsino, and P. Cicuta, Proc. Natl. Acad. Sci. U.S.A. 107, 7669 (2010).

[14] G. M. Cicuta, E. Onofri, M.C. Lagomarsino, and P. Cicuta, Phys. Rev. E 85, 016203 (2012).

[15] M. Reichert and H. Stark, Eur. Phys. J. E 17, 493 (2005).

[16] M. C. Lagomarsino, P. Jona, and B. Bassetti, Phys. Rev. E 68, 021908 (2003).

[17] A. Vilfan and F. Jülicher, Phys. Rev. Lett. 96, 058102 (2006).

[18] P. Lenz and A. Ryskin, Phys. Biol. 3, 285 (2006).

[19] C. Wollin and H. Stark, Eur. Phys. J. E 34, 42 (2011).

[20] N. Uchida and R. Golestanian, Phys. Rev. Lett. 104, 178103 (2010).

[21] N. Uchida and R. Golestanian, Phys. Rev. Lett. 106, 058104 (2011); Eur. Phys. J. E (in press).

[22] T. Niedermayer, B. Eckhardt, and P. Lenz, Chaos, 18, 037128 (2008).

[23] D. M. Woolley, R. F. Crockett, W. D. I. Groom, and S. G. Revell, J. Exp. Biol. 212, 2215 (2009).
[24] M. Polin, I. Tuval, K. Drescher, J.P.Gollub, and R.E. Goldstein, Science 325, 487 (2009); R. E. Goldstein, M. Polin, and I. Tuval, Phys. Rev. Lett. 103, 168103 (2009); 107, 148103 (2011).

[25] D. L. Kirk, Volvox: Molecular-Genetic Origins of Multicellularity and Cellular Differentiation (Cambridge University Press, Cambridge, England, 1998).

[26] K. Drescher, K. C. Leptos, I. Tuval, T. Ishikawa, T. J. Pedley, and R.E. Goldstein, Phys. Rev. Lett. 102, 168101 (2009).

[27] D. L. Kirk and M. M. Kirk, Dev. Biol. 96, 493 (1983).

[28] M. Raffel, C.E. Willert, S. T. Wereley, and J. Kompenhans, Particle Image Velocimetry (Springer, Berlin, 2007), 2nd ed.

[29] See Supplemental Material at http://link.aps.org/ supplemental/10.1103/PhysRevLett.109.268102 for a video of the time-dependent flow field and the timeaveraged velocity profile for a representative colony.

[30] S. Kim and S. J. Karrila, Microhydrodynamics (Dover Publications, Mineola, NY, 1991).

[31] A. Pikovsky, M. Rosenblum, and J. Kurths, Synchronization: A Universal Concept in Nonlinear Sciences (Cambridge University Press, Cambridge, England, 2003).

[32] J. R. Blake, Math. Proc. Cambridge Philos. Soc. 70, 303 (1971).

[33] H. Sakaguchi, S. Shinomoto, and Y. Kuramoto, Prog. Theor. Phys. 79, 1069 (1988). 